\title{
Isolation, characterization, and evaluation of Bacillus thuringiensis isolated from cow milk
}

\author{
Chang-Hee Kweon ${ }^{1, *, \dagger}$, Sang-Yoon Choi ${ }^{2, \dagger}$, Hyog-Young Kwon ${ }^{2}$, Eun-Hye Kim ${ }^{2}$, Hyun-Mi Kang ${ }^{1}$, Jin-San Moon ${ }^{1}$, \\ Geum-Chag Jang ${ }^{1}$, Hee-Soo Lee ${ }^{1}$, Seung-Won Kang ${ }^{1}$, Jong-man Kim ${ }^{1}$, Suhkneung Pyo ${ }^{2}$, Dong-Kwon Rhee ${ }^{2, *}$ \\ ${ }^{1}$ Animal, Plant and Fisheries Quarantine and Inspection Agency (QIA), Anyang 430-757, Korea \\ ${ }^{2}$ School of Pharmacy, SungKyunKwan University, Suwon 441-746, Korea \\ (Received: April 19, 2012; Revised: July 2, 2012; Accepted: August 14, 2012)
}

\begin{abstract}
Probiotics colonize the intestines and exert an antibacterial effect on pathogens. Therefore, probiotics could be used as a preventive agent against lethal infections. To isolate probiotic microorganisms, 116 bacterial strains were isolated from healthy cow's milk and were subjected to Gram-stain, morphology and biochemical analyses, Vitek analysis, and 16S rRNA analysis. One of the strains identified as Bacillus (B.) thuringiensis 87 was found to grow very well at $\mathrm{pH} 4.0 \sim 7.0$ and to be resistant to high concentrations of bile salts $(0.3 \sim 0.9 \% \mathrm{w} / \mathrm{v})$. B. thuringiensis was susceptible to the antibiotics used in the treatment of bovine mastitis, yet it exhibited an antimicrobial effect against Staphylococcus $(S$.) aureus 305. Moreover, it protected mice from experimental lethal infections of E. coli O55, Salmonella typhimurium $01 \mathrm{D}$, and $S$. aureus 305 through a significant induction of interferon- $\gamma$, even at four-week post-administration of $B$. thuringiensis. Although oral administration of $B$. thuringiensis 87 did not provide significant protection against these lethal challenges, these results suggest that $B$. thuringiensis 87 could be a feasible candidate as a probiotic strain.
\end{abstract}

Keywords : Bacillus thuringiensis, interferon- $\gamma$, probiotics

\section{Introduction}

Probiotics are live microorganisms that exhibit beneficial effects on a host when ingested in sufficient amounts [2]. Generally, probiotics improve symptoms caused by intestinal bacterial fermentation. They influence microflora congestion on the intestinal wall and produce various products that aid in the absorption and utilization of ingested nutrients [24]. They also generate substances that neutralize toxins produced by bacteria and inhibit pathogen growth and proliferation [9]. Probiotics include Bacillus, Enterococcus, Streptococcus, Lactobacillus species ( $s p$ ), and yeast [7] and are commonly found in the fermentation process of dairy products, crude oil, and in the intestines of animals and humans. For example, Lactobacillus sp maintains an acidic $\mathrm{pH}$ in the intestines, which inhibits the growth of intestinal pathogens such as E. coli, Clostridium sp, or bacteria causing diarrhea, and supports a healthy intestinal microflora. It has been suggested that probiotics may be useful as therapeutics $[1,10$, 17, 20, 23, 26].

Probiotics might be used to minimize the need to use antibiotics in feed. Antibiotics are commonly added into feed in the livestock industry to prevent diseases caused by pathogenic microorganisms, thus increasing the economic productivity of livestock. However, because of the emergence of antibiotic-resistant bacteria and antibiotic residuals that have recently emerged as a serious problem, the use of antibiotics tends to be regulated $[6,8,22]$. Therefore, the use of antibiotics is only encouraged for the treatment of diseases, with the use of probiotics being suggested for prevention or convalescence of diseases.

Probiotics in Bacillus (B.) species, including B. subtilis, B. licheniformis, and $B$. coagulance, are known to directly and indirectly prevent pathogenic damage through antagonistic action or secretion $[12,14]$. In addition, probiotic microorganisms added to the feed can reach the intestine, where they can attach to the walls of epithelial cells, thus preventing other harmful pathogens from colonizing and improving the health of the animal. The aims of this study were to isolate the potential candidate strains from healthy cow's milk and to identify possible probiotic strains. Among the 116 bacterial strains isolated, $B$. thuringiensis 87 showed the best probiotic characteristics, as demonstrated by acid- and bile saltresistances and a protective effect. These features imply for

*Corresponding authors

Tel: +82-31-467-1753, Fax: +82-31-467-1797

E-mail: Kweonch@korea.kr (CH Kweon),dkrhee@skku.edu (DK Rhee)

These first two authors contributed equally to this work. 
the first time that $B$. thuringiensis could be a feasible probiotic of livestock through the elicitation of nonspecific immunity for the prevention of animal diseases.

\section{Materials and Methods}

\section{Bacterial strains, cultures, and animals}

B. subtilis 1024 and Staphylococcus (S.) aureus 305 were purchased from American Type Culture Collection (ATCC1024 and ATCC305, respectively, USA). Streptococcus (S.) pneumoniae encapsulated type 2 strain D39 (NCTC7466) was cultured in brain heart infusion (BHI) broth or on BHI agar (Oxoid, UK) as described previously [18]. Commercially available B. subtilis, B. coagulans, and B. mesentericus strains were donated by Handong and Sungwon (Korea) for use as references. E. coli 055 , E. coli 21 , and Salmonella $(S$.) typhimurium 01D strains were isolated from pigs and cows in South Korea at the Animal, Plant and Fisheries Quarantine and Inspection Agency with intestinal diseases including diarrhea.

All Bacillus sp strains, S. aureus ATCC305, S. pneumoniae $\mathrm{D} 39$, and $S$. typhimurium $01 \mathrm{D}$ were grown in BHI broth or on BHI agar, and E. coli 055 was grown in Luria-Bertani (LB) broth or on LB agar. All inoculated broth or agar plates were incubated at $37^{\circ} \mathrm{C}$, and colonies were counted after $18 \mathrm{~h}$ incubation. CD1 mice were purchased from Samtako (Korea).

\section{Isolation and identification of microorganisms from healthy cow's milk}

In our search for an effective probiotic, microbial strains were collected from fresh raw milk from 35 dairy farms at 22 geographically different locations from March to August 2001 in South Korea. To avoid contamination, the first part of the milk was not collected. Microorganisms were isolated from milk only when the somatic cell count was less than 30,000 according to an automatic somatic cell counter (Fossomatic series 4000; Foss Electric, Denmark). Raw milk was kept on ice after sampling and was directly processed for determination of total colony. For total colony count, $1 \mathrm{~mL}$ of the sample suspension was diluted decimally and was pour plated in duplicate directly on milk plate count agar (Oxoid). All plates were incubated at $30^{\circ} \mathrm{C}$ for $72 \mathrm{~h}$ prior to count colonies. For isolation of potential probiotics, and after cooling on ice, $1 \mathrm{~mL}$ was immediately spread plated in duplicate onto petri dishes containing BHI broth supplemented with filtersterilized vitamin $\mathrm{B}_{12}$ (1 $\mathrm{mg} \mathrm{liter}^{-1}$; Sigma, USA), pH 6.8 and bacteriological agar no. 1 (15 g liter ${ }^{-1}$; Oxoid). All visibly different colonies were picked off $(n=116)$. Pure cultures of these isolates were stored at $-80^{\circ} \mathrm{C}$. After the samples were selected, they were further incubated on a blood agar plate at $37^{\circ} \mathrm{C}$ for 16 h. Initially, 116 strains comprising Bacillus, Enterococcus, and Lactobacillus were isolated from fresh milk and were further cultured on BHI agar, and their morphologies and cultural and physiological characteristics including blood agglutination, Gram staining, were carried out according to Bergey's manual. Also antibiotic resistance was determined by disc diffusion method as suggested by guidelines of the Clinical and Laboratory Standard Institute [5]. The bacterial strains were also screened using the GPI card of the Vitek system (BioMerieux, USA).

\section{Identification of Bacillus strains}

To further identify the Bacillus strains, the carbohydrate utilization pattern and nucleotide sequence of $16 \mathrm{~S}$ ribosomal RNA were determined via PCR methods using conserved sequences. The conserved 16S rRNA sequences in Bacillus strains used for amplification were 16S-27F (5'AGR GTT TGA TCM TGG CTC AG 3') and 16S-1492R (5' GGY TAC CTT GTT ACG ACT T 3') [19]. To further identify the Bacillus species, conserved 16S rRNA sequences were used to amplify the rDNA using an $\mathrm{ABI}$ thermal cycler; forward primers 16S-F23 (5'GGC GGC GTG CCT AAT ACA TGC AAG TCG3') and 16S-F310 (5'CGG CCC AGA CTC CTA CGG GAG GCA GCA3') and reverse primer 16S-R770 (5'GCG TGG ACT ACC AGG GTA TCT AAT CC3') [19]. Amplified PCR products were subsequently separated using gel electrophoresis in a $1.2 \%$ agarose gel, were cut from the agarose gel and used for nucleotide sequence determination. Nucleotide sequences were determined using the above primers and a BigDye terminator cycle sequencing kit (Applied Biosystems, USA) in an automatic DNA sequencer (Applied Biosystems Model 310) according to the manufacturer's instructions. The nucleotide sequence was deposited into GeneBank (Accession no. HQ845263). Using the BLASTN tool, the nucleotide sequences were compared against those in the National Center for Biotechnology Information GenBank. The identified strain was deposited into the Korean Agricultural Culture Collection (KACC 91098).

\section{Screening for $\mathbf{p H}-$ and bile-salt-tolerant strains}

BHI broth adjusted to $\mathrm{pH} 1 \sim 7$ with $\mathrm{HCl}$ (Sigma) was used to culture all of the Bacillus strains, which were then incubated at $37^{\circ} \mathrm{C}$ for $18 \mathrm{~h}$ followed by measurement of absorbance at $620 \mathrm{~nm}$. Bile salt tolerance was determined in $\mathrm{BHI}$ broth after adding bile salt (Difco, USA) to a final concentration of $0.3 \%, 0.6 \%$, or $0.9 \%$. Then, the Bacillus strains were cultured at $37^{\circ} \mathrm{C}$ for $16 \mathrm{~h}$, and the absorbance at $620 \mathrm{~nm}$ was measured to assess growth.

\section{Antimicrobial resistance testing}

Antimicrobial resistance was determined by disc diffusion method according to the guidelines of the Clinical and Laboratory Standard Institute [5]. Antibiotic discs (BD, USA) were placed onto a lawn of freshly plated bacteria on the Muller-Hinton agar containing 3\% (v/v) sheep blood, and antimicrobial resistance was determined by measuring the diameter of the inhibition zone after incubation of the plate at $37^{\circ} \mathrm{C}$ for $16 \mathrm{~h}$. The antibiotics in each disc included ampicillin $(10 \mu \mathrm{g})$, amikacin $(30 \mu \mathrm{g})$, cephalothin $(30 \mu \mathrm{g})$, cloxacillin $(1 \mu \mathrm{g})$, erythromycin $(15 \mu \mathrm{g})$, enrofloxacin $(30 \mu \mathrm{g})$, gentamy- 
cin $(10 \mu \mathrm{g})$, kanamycin $(30 \mu \mathrm{g})$, lincomycin $(2 \mu \mathrm{g})$, neomycin $(30 \mu \mathrm{g})$, oxacillin $(1 \mu \mathrm{g})$, penicillin $\mathrm{G}(10 \mathrm{U})$, streptomycin $(10 \mu \mathrm{g})$, tetracycline $(30 \mu \mathrm{g})$, or vancomycin $(30 \mu \mathrm{g})$.

\section{Antagonism test}

To determine the in vitro antagonistic effects of the probiotic strains against $S$. aureus, both probiotic strain and $S$. aureus ATCC305 strain were cultured overnight, and the next day they were inoculated into a fresh medium until they reach mid-log phase. Subsequently, probiotic strain was added into culture of $S$. aureus ATCC305 to a final volume of $1 \%$ (1/100 dilution). Then the mixed culture was incubated at $37^{\circ} \mathrm{C}$ for $1,3,6$, or $24 \mathrm{~h}$, and viable cell numbers of S. aureus were determined after plating on Baird-Parker agar containing $5 \%$ egg yolk.

\section{In vivo challenge experiments}

To determine the presence of a protective effect against virulent pathogens in the probiotic strains in vivo, CD1 mice (four-week-old males) were injected intraperitoneally (i.p) with $100 \mu \mathrm{L}$ of $B$. thuringiensis 87 (approximately $1 \times 10^{8}$ CFU suspended in PBS). This experiment was also conducted to identify the potential risk of toxigenicity and hypersensitivity of strain.

Three days post-administration, each mouse was challenged i.p. with $100 \mu \mathrm{L}$ of a lethal dose of virulent pathogen $\left(2.5 \times 10^{9} \mathrm{CFU}\right.$ of $E$. coli $055,2 \times 10^{8} \mathrm{CFU}$ of $S$. aureus 305 , or $7.3 \times 10^{6} \mathrm{CFU}$ of $S$. typhimurium $\left.01 \mathrm{D}\right)$ suspended in sterile PBS. In the case of the $S$. aureus challenge, one week post-administration of $B$. thuringiensis $87,100 \mu \mathrm{L}$ of $S$. aureus ATCC305 $\left(2 \times 10^{8} \mathrm{CFU} / \mathrm{mL}\right.$ in PBS) was injected i.p. To differentiate $S$. aureus from Bacillus, S. aureus was selected from Baird-Parker agar containing 5\% egg yolk. For each strain, at least two experiments were performed. Survival of the mice was checked every day.

\section{Determination of interferon- $\gamma$}

The interferon (IFN)- $\gamma$ level in serum was determined using an ELISA kit (BD) according to manufacturer's instructions as follows. One hundred $\mu \mathrm{L}$ of serum was added into microtiter plate well, and primary antibody diluted to $2,000 \mathrm{pg} / \mathrm{mL}$ was added. After incubation of the mixture at room temperature for $2 \mathrm{~h}$, the titer plate was washed 5 times with PBS. Subsequently, secondary antibody diluted 250 fold was added and incubated for $30 \mathrm{~min}$ at room temperature. After washing 5 times, detector solution was added and incubated $1 \mathrm{~h}$ further. The titer plate was washed 7 times and substrate $(3,3$ ',5,5'-tetramethyl benzidine: TMB) was added and incubated at room temperature for $30 \mathrm{~min}$ in dark place. To stop the reaction, stop solution was added, and OD at $450 \mathrm{~nm}$ was determined.

\section{Statistics}

For statistical analysis, the OD differences among groups were calculated using the one-way ANOVA Newman-Keuls test. Statistical differences between the group medians were analyzed using the Mann-Whitney U test (two-tailed unpaired). ELISA data were expressed as the average of triplicate wells \pm SEM. Statistically significant differences were defined as $p$ $<0.05$.

\section{Results}

Identification of $B$. thuringiensis from bacterial strains isolated from fresh cow milk

In total, 116 bacterial strains were isolated from fresh raw milk, which was chosen only when it contained less than 30,000 somatic cells. Gram staining and morphological observation showed the isolated strains were either Grampositive bacilli or cocci. The strains were also identified using the Vitek system (bioMrieux, France), which indicated the presence of Bacillus, Enterococcus, and Lactobacillus species (data not presented).

To further identify the strain, sequencing of $16 \mathrm{~S}$ rRNA nucleotide was performed. Results showed 99\% homology with the $B$. thuringiensis species (Table 1), and the strain was named B. thuringiensis 87.

\section{pH and bile salt resistance of $B$. thuringiensis 87}

First, $\mathrm{pH}$ sensitivity was studied over a range of $\mathrm{pH}$ from 1 to 7 and B. thuringiensis 87 as well as other Bacillus. Results showed that $B$. thuringiensis 87 , as well as other Bacillus strains such as B. subtilis, B. coagulans, and B. mesentericus, grew at $\mathrm{pH} 5$ or above but did not grow in conditions less than $\mathrm{pH} 4$ (Fig. 1A).

To assess bile salt tolerance, the growth rates of a variety of Bacillus strains were determined at different concentrations of bile salt in BHI broth. The results showed that $B$. thuringiensis 87 and several other Bacillus strains survived at bile salt proportions of $0.3 \%$ to $0.9 \%$. At a $0.9 \%$ bile salt concentration, the absorbances of $B$. thuringiensis 87 and $B$. coagulans were similar, whereas those of commercially available probiotics B. subtilis (A), B. subtilis (B) and B. mesentericus were lower. $B$. subtilis had a low survival rate throughout the various concentrations of bile salt. These data suggest that $B$. thuringiensis 87 is resistant to a high bile salt

Table 1. Identification of Bacillus thuriengiensis 87 via DNA sequence comparison in GenBank

\begin{tabular}{ccccc}
\hline \hline & $\begin{array}{c}\text { Genebank } \\
\text { Accession No. }\end{array}$ & Identity & Total length & Homology region \\
\hline Bacillus thuringiensis str. Al Hakam & CP000485.1 & $1508 / 1512(99 \%)$ & 5257091 & $258211-259722$ \\
Bacillus thuringiensis serovar konkukian str & AE017355.1 & $1505 / 1510(99 \%)$ & 5237682 & $9320-10829$ \\
\hline
\end{tabular}


A

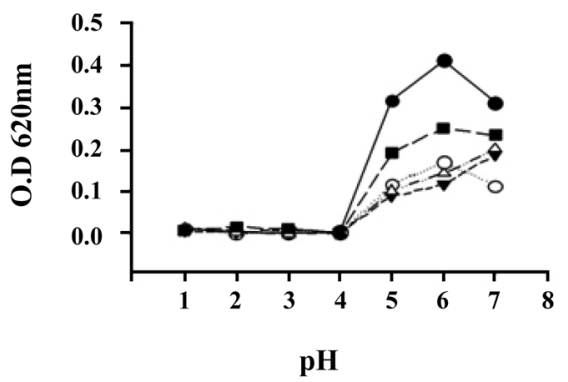

B
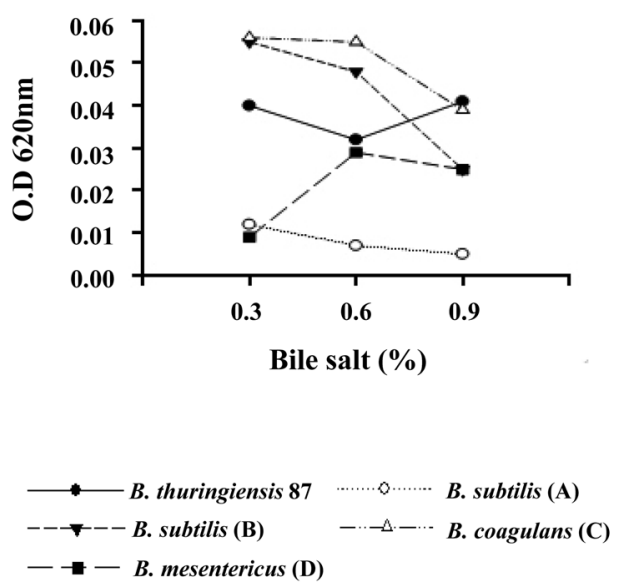

Fig. 1. Effects of $\mathrm{pH}(\mathrm{A})$ and bile salts (B) on the growths of various Bacillus species at $37^{\circ} \mathrm{C}$. To check $\mathrm{pH}$ and bile-salt resistance, the growth of Bacillus species in BHI broth was determined by $\mathrm{OD}$ at $620 \mathrm{~nm}$ after adjusting the culture media to the specific $\mathrm{pH}$ or salt concentration.

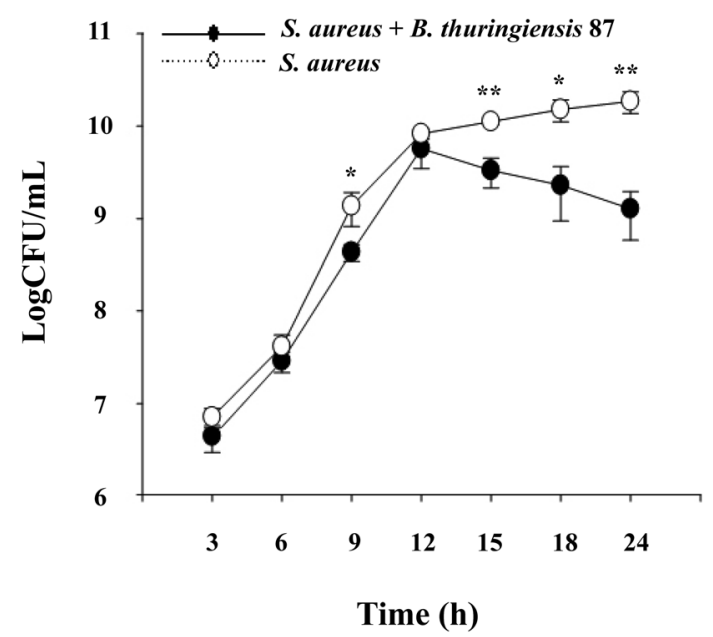

Fig. 2. Antimicrobial activity of Bacillus (B.) thuringiensis 87 against pathogenic Staphylococcus (S.) aureus 305. To determine their antimicrobial activities, $B$. thuringiensis 87 was inoculated into $S$. aureus 305 cultures to a final volume of $1 \%$ (1/ 100 dilution). Then the mixed culture was incubated, and a viable cell number of $S$. aureus was selected by plating on BairdParker agar containing 5\% egg yolk. concentration (Fig. 1B).

\section{In vitro antimicrobial activity of $B$. thuringiensis 87}

Results showed that the growth of the pathogenic strain was significantly decreased after 6 and $24 \mathrm{~h}$ of co-culture compared to that of the control group, indicating that $B$. thuringiensis 87 had a detectable antibiotic effect (Fig. 2). Also antibiotic effect of $B$. thuringiensis 87 was the same or better than those of the other commercially available strains (data not shown). This result indicates that $B$. thuringiensis 87 has desirable antibacterial effects against the pathogenic $S$. aureus strain.

\section{Antibiotic resistance of $B$. thuringiensis 87}

Since antibiotic-susceptible probiotics are preferred, we determined the antibiotic resistance of $B$. thuringiensis 87 to several antibiotics. Since the guidelines for antibiotic resistance are only set for human clinical isolates and guidelines for antibiotic resistance for probiotic organisms are not available, we could not define true antibiotic resistance for $B$. thuringiensis 87 . However, the results showed that $B$. thuringiensis 87 had the same resistance pattern as did the other commercially available reference Bacillus strains used in this study. These results indicate that $B$. thuringiensis 87 was susceptible to antibiotics such as ampicillin, amikacin, cephalothin, cloxacillin, erythromycin, enrofloxacin, gentamycin, kanamycin, lincomycin, neomycin, oxacillin, penicillin G, streptomycin, tetracycline, and vancomycin (Supplementary Table 1).

\section{Virulence attenuation of $\boldsymbol{B}$. thuringiensis 87}

Invasion of pathogenic bacteria from the intestine into the blood can lead to high morbidity, septic shock, and death. Since B. thuringiensis 87 has a similar antimicrobial effect to those of other commercially available Bacillus species, the virulence of $B$. thuringiensis 87 was examined using the septicemia model. B. thuringiensis $87\left(7 \times 10^{7} \mathrm{CFU}\right)$ or S. pneumoniae D39 $\left(1 \times 10^{4} \mathrm{CFU}\right)$ was inoculated intraperitoneally (i.p) into mice, and survival time was determined. The results showed that $B$. thuringiensis 87 , along with PBS as the negative control, caused no mice mortalities within seven days (Fig. 3A), demonstrating that the virulence of B. thuringenisis 87 was absent or significantly attenuated and suggesting that $B$. thuringenisis 87 might be a highly feasible candidate as a probiotic.

Protection from lethal infections by $\boldsymbol{B}$. thuringiensis 87 To check the probiotic effect of the strain in question, groups of ten or 11 mice were inoculated i.p with $7 \times 10^{7}$ CFU of B. thuringenisis 87. Three days post-infection, mice were challenged i.p with either $2.5 \times 10^{9} \mathrm{CFU}$ of pathogenic E. coli 055 or $7.3 \times 10^{6} \mathrm{CFU}$ of pathogenic S. typhimurium $01 D$. Ninety percent of mice were killed within six days when challenged with E. coli 055 and within two days when challenged with $S$. typhimurium 01D. In contrast, mice that received B. thuringenisis 87 prior to challenge with E. coli 
Supplementary Table 1. Antibiotic resistances of Bacillus (B.) thuringiensis 87 and other Bacillus species

\begin{tabular}{|c|c|c|c|c|c|c|c|c|c|c|c|c|c|c|}
\hline \multirow{2}{*}{ Strain } & \multicolumn{14}{|c|}{ Antibiotic resistance, growth inhibition diameter $(\mathrm{mm})$} \\
\hline & $\mathrm{AN}$ & $\mathrm{N}$ & $\mathrm{S}$ & $\mathrm{K}$ & TE & $\mathrm{P}$ & $\mathrm{E}$ & ENR & VA & GM & OX & $\mathrm{AM}$ & $\mathrm{CF}$ & $\mathrm{CX}$ \\
\hline B. thuringiensis 87 & 21 & 21 & 24 & 24 & 28 & 18 & 30 & 28 & 22 & 22 & - & 19 & 21 & - \\
\hline B. subtilis ${ }^{a}$ & 27 & 25 & 20 & 28 & 22 & 13 & 12 & $\mathrm{ND}^{*}$ & 25 & 32 & 11 & 16 & 25 & 15 \\
\hline B. subtilis ${ }^{b}$ & 30 & 23 & 20 & 11 & 25 & 33 & 30 & ND & 28 & 30 & 24 & 32 & 42 & 15 \\
\hline B. coagulans ${ }^{c}$ & 26 & 21 & 18 & 24 & 19 & 30 & 25 & ND & 20 & 26 & 23 & 30 & 40 & 22 \\
\hline B. mesentericus ${ }^{d}$ & 22 & 23 & 20 & 30 & 19 & 27 & 27 & ND & 20 & 26 & 17 & 25 & 40 & 17 \\
\hline
\end{tabular}

AM: ampicillin $(10 \mu \mathrm{g}), \mathrm{AN}$ : amikacin $(30 \mu \mathrm{g}), \mathrm{CF}$ : cephalothin $(30 \mu \mathrm{g}), \mathrm{CX}$ : cloxacillin $(1 \mu \mathrm{g})$, E: erythromycin $(15 \mu \mathrm{g})$, ENR: enrofloxacin $(30 \mu \mathrm{g})$, GM: gentamycin $(10 \mu \mathrm{g}), \mathrm{K}$ : kanamycin $(30 \mu \mathrm{g})$, L: lincomycin $(2 \mu \mathrm{g})$, N: neomycin $(30 \mu \mathrm{g}), \mathrm{OX}$ : oxacillin $(1 \mu \mathrm{g})$, P: penicillin G (10 U), S: streptomycin (10 $\mu \mathrm{g})$, TE: tetracycline $(30 \mu \mathrm{g})$, VA: vancomycin $(30 \mu \mathrm{g}){ }^{\mathrm{a}, \mathrm{b}, \mathrm{d}, \mathrm{d}}$ Commercially available products. ${ }^{*} \mathrm{ND}$ : Not determined.

A

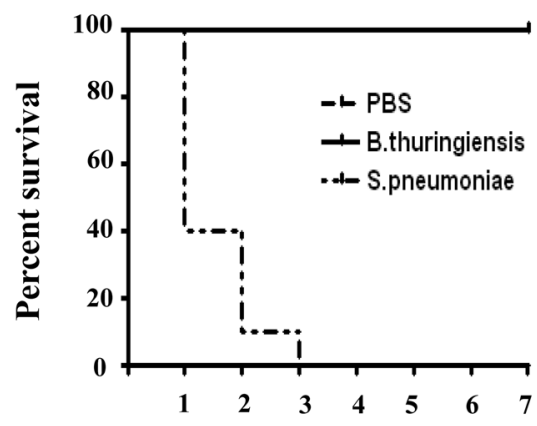

Days

C

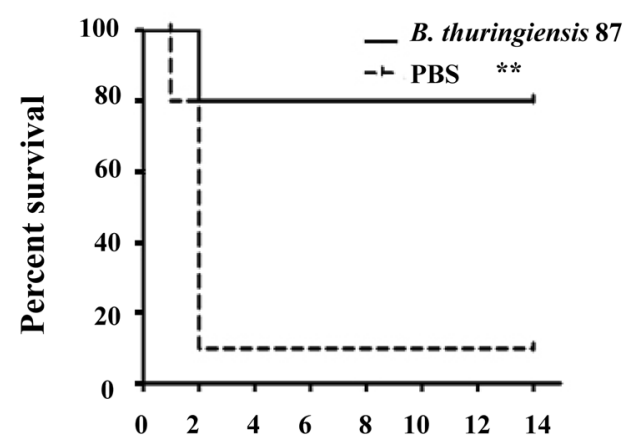

Days
B

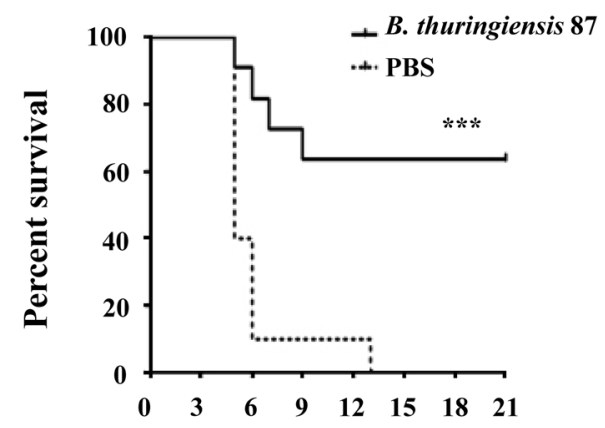

Days

D

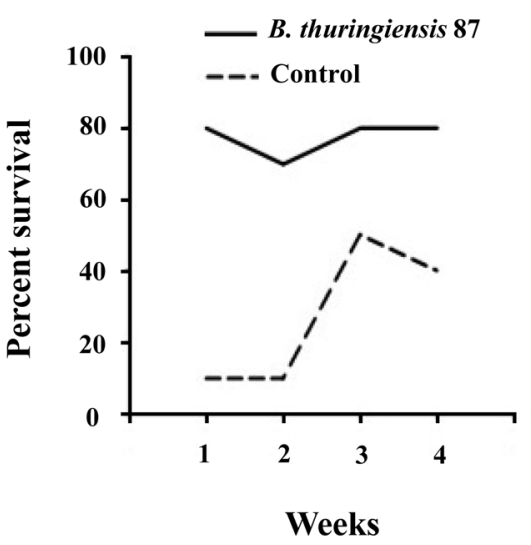

Fig. 3. Probiotic effects of B. thuringiensis 87 against pathogens. (A) Virulence attenuation of B. thuringiensis 87 in vivo after intraperitoneal inoculation. A group of 10 mice was inoculated with $7 \times 10^{7} \mathrm{CFU}$ of B. thuringiensis $87,1 \times 10^{4} \mathrm{CFU}$ of $S$. pneumoniae D39, or PBS as a control. (B, C) Protection from E. coli 055 and S. typhimurium $01 \mathrm{D}$ challenges. Groups of ten or 11 mice were intraperitoneally inoculated with $7 \times 10^{7} \mathrm{CFU}$ of $B$. thuringenisis 87 , three days prior to an intraperitoneal inoculation with either $2.5 \times 10^{9}$ CFU of E. coli 055 (B) or $7.3 \times 10^{6} \mathrm{CFU}$ of $S$. typhimurium $01 \mathrm{D}$ (C). (D) Long-term protection by B. thuringenisis 87 against $S$. aureus 305 challenge. Forty mice were intraperitoneally immunized with $B$. thuringenisis $87\left(1.1 \times 10^{8} \mathrm{CFU}\right)$ and then divided into 4 groups, and subsequently challenged by $S$. aureus $305\left(2 \times 10^{8} \mathrm{CFU}\right) 1,2,3$, and 4 weeks post-inoculation. The survival was recorded every day and was analyzed using the Prism survival test $\left(p=0.0016,{ }^{* * *} p<0.01,{ }^{* * *} p<0.001\right)$. 
A

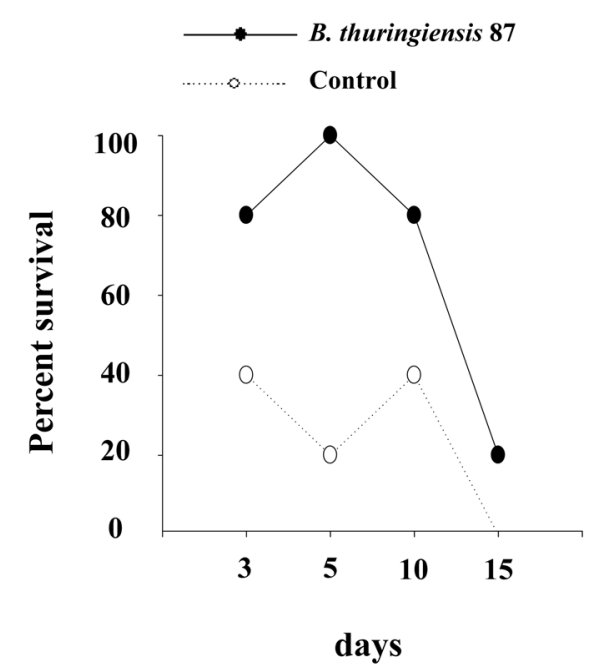

B

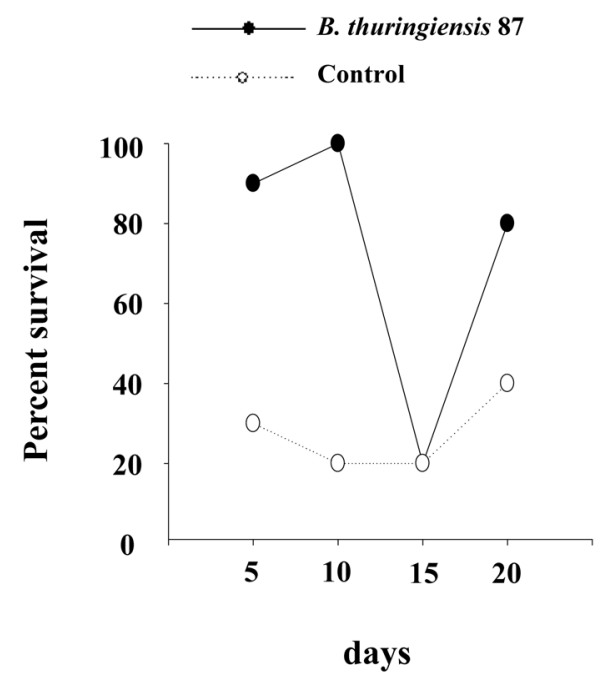

Fig. 4. Protection from lethal challenge with E. coli 21 or $S$. aureus 305 by heat-killed B. thuringiensis $87.1 .1 \times 10^{8} \mathrm{CFU}$ of the heatkilled B. thuringiensis 87 was inoculated prior to lethal challenge. For lethal challenge, either S. aureus $305\left(2 \times 10^{8}\right.$, panel A) or E. coli $21\left(2.5 \times 10^{9} \mathrm{CFU}\right.$, panel B) was inoculated i.p. Each group consisted of five mice.

055 showed a $64 \%$ survival rate 21 days post-challenge $(p<$ 0.001 ; Fig. 3B). Consistently, mice given B. thuringenisis prior to challenge with $S$. typhimurium $01 \mathrm{D}$ showed an $80 \%$ survival rate 14 days post-challenge ( $p<0.01$; Fig. $3 \mathrm{C}$ ). These results demonstrate that $B$. thuringenisis 87 could serve as a probiotic to protect animals from invasive infection by pathogenic bacteria.

To further corroborate the probiotic effect of $B$. thuringenisis 87 against Gram-positive pathogens, S. aureus 305 was used as the challenge organism. Before determination of the probiotic effect of $B$. thuringenisis 87, the virulence of the $S$. aureus 305 strain was determined after i.p inoculation. When $8 \times 10^{8} \mathrm{CFU}$ or $4 \times 10^{8} \mathrm{CFU}$ of $S$. aureus 305 was inoculated into 5 mice i.p, all five mice were dead within a day, whereas i.p inoculation of $2 \times 10^{8} \mathrm{CFU}$ of $S$. aureus 305 resulted in the death of four mice within a day (data not shown). Therefore, to determine the probiotic effect of $B$. thuringenisis 87 , we inoculated $B$. thuringenisis 87 i.p, and then, 1-, 2-, 3-, and 4-weeks post-inoculation, mice were challenged i.p with $2 \times 10^{8} \mathrm{CFU}$ of $S$. aureus 305 . One- to four-weeks post-administration, the mice that received $B$. thuringenisis 87 showed significantly increased survival rates compared to those of the control group. The average survival rate of the $B$. thuringenisis 87 pre-administered group was $77.5 \%$, whereas that of the control group was $27.5 \%$ (Fig. 3D). These data confirm that B. thuringenisis 87 inoculation could be used for probiotic protection against invasive virulent pathogens in animals.

Although inoculation of sonicated lysate of $7 \times 10^{7} \mathrm{CFU} B$. thuringenisis 87 did not provide protection from $S$. aureus 305 three days post-inoculation (data not shown), inoculation of heat-killed B. thuringenisis 87 provided protection from $S$. aureus 305 three to ten days post-inoculation (Fig. 4A). Consistently, heat-killed B. thuringenisis 87 provided protection from $E$. coli 21 five to ten days post-inoculation (Fig. 4B). These results demonstrate that live or dead B. thuringenisis 87 , but not the lysates, can be used to prevent invasive pathogen infection.

To corroborate the probiotic effect of the dead B. thuringenisis 87, it was i.p. inoculated twice over a one-week interval and was challenged one week after the last inoculation with either $S$. aureus 305 or $E$. coli 21 . When mice were inoculated twice with heat-killed B. thuringiensis 87 followed by $S$. aureus 305 challenge, we observed a $100 \%$ survival rate, whereas the control without $B$. thuringiensis 87 inoculation showed no survival (data not shown). Consistent with this, two inoculations of heat-killed B. thuringiensis 87 followed by $E$. coli 21 challenge provided $80 \%$ survival, whereas the control group showed only $10 \%$ survival (data not shown). These results indicate that inoculation with heatkilled $B$. thuringenisis 87 could be useful for protection against invasive pathogen infection.

\section{Induction of IFN- $\gamma$ by administration of $B$. thuring- iensis 87}

To further investigate the underlying mechanism of the protective effect of $B$. thuringenisis 87 , the strain was inoculated i.p. and the viability of B. thuringenisis 87 and IFN- $\gamma$ levels in blood were determined two and four weeks postadministration. Although no viable cells were detected at these times (data not shown), IFN- $\gamma$ was increased 7.1-fold significantly after 2 weeks post-administration of $B$. thuringenisis 87 (Fig. 5). Moreover, three to 20 days post-inoculation with heat-killed $B$. thuringenisis 87 , the level of IFN- $\gamma$ 


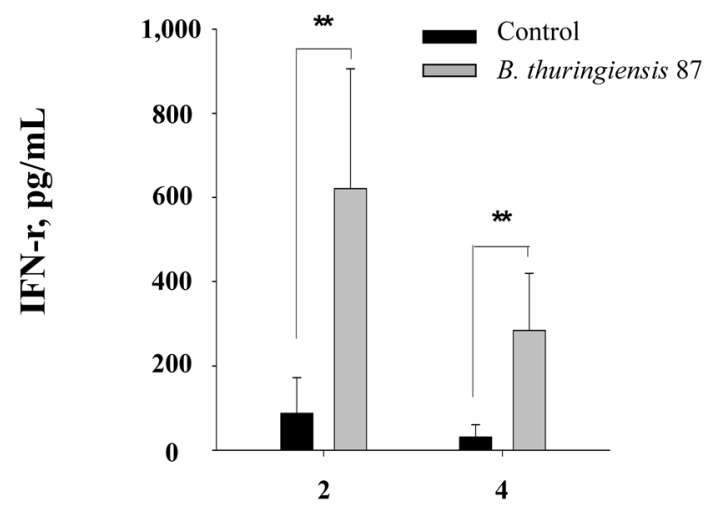

Time (weeks)

Fig. 5. Induction of interferon (IFN)- $\gamma$ by B. thuringiensis 87 injection. $1.1 \times 10^{8} \mathrm{CFU}$ of $B$. thuringiensis 87 was inoculated i.p. Two and four weeks post-administration of $B$. thuringiensis 87 , a blood sample was taken and the IFN- $\gamma$ level was determined using ELISA. Three mice per group were used.

was maintained at a higher level than that of the control group (data not shown), indicating that administration of $B$. thuringenisis 87 could activate the immune system and protect the host from invading pathogens.

\section{Discussion}

Some Bacillus species posses probiotic characteristics such as resistance to strong acids from stomach and bile acids; tolerance to heat, acids, and humidity; and formation of polypeptide antibiotics like bacitracin [12]. B. thuringiensis, the spore-forming bacterium, is well known as a bioinsecticide that controls plant diseases. The strain produces several plasmid-encoded Cry proteins in large quantities during sporulation and which are packaged into intracellular crystal inclusions. Inclusions ingested by insect larvae are solubilized and converted to active toxins in the midgut. Subsequently, toxins are inserted into the membrane, resulting in the formation of cation-selective channels and lethality due to osmotic lysis [3, 4, 25]. Moreover, in the mammalian intestinal tract, $B$. thuringiensis has been shown to germinate and transfer DNA [28]. However, the role of B. thuringiensis as a probiotic has not received much attention. This is the first study to isolate and characterize B. thuringiensis 87 from fresh cow milk and to provide evidence that it is a probiotic strain.

In this study we demonstrate that the B. thuringiensis 87 isolated has acid- and bile-resistance, broad carbohydrate utilization, and growth inhibitory effects against harmful bacteria. Moreover, we demonstrate the non-toxicity of $B$. thuringiensis 87 in vivo and its protective effect against lethal pathogenic infections including $S$. aureus 305, E. coli 21 and $S$. typhimurium 01D. Although heat-killed B. thuringiensis 87 could protect mice from lethal challenges, live $B$. thuring- iensis 87 has stronger protective effects. Since B. thuringiensis 87 can protect against lethal septicemia even four weeks post-inoculation, it is highly feasible that it could be an excellent probiotic for the prevention of lethal diseases due to invasive pathogens in animals. Upon infection with pathogens, specific pathogen-associated molecular patterns are recognized by phagocyte receptors, resulting in the triggering of the type- 1 early cytokine (IFN- $\gamma$, interleukin 12 , interleukin 23 , interleukin 17) signaling pathway. Activation of this pathway initiates microbicidal mechanisms in phagocytes [27].

The protective effects of $B$. thuringenisis 87 can be explained by 1) the antimicrobial and/or microcidal activity of $B$. thuringenisis 87,2 ) activation of the immune system to result in pathogen removal, and 3) colonization in the host. The antimicrobial activity of $B$. thuringenisis 87 was detected. Moreover, i.p. injections of B. thuringiensis 87 provided protection against lethal challenges, although oral administration of $B$. thuringiensis 87 did not provide protection against lethal challenge (data not shown). Since administration of $B$. thuringiensis 87 significantly induced IFN- $\gamma$, a key cytokine that induces mouse and human macrophages to kill pathogens through both oxidative burst and nonoxidative mechanisms $[13,15,16,21]$, and mice deficient for IFN- $\gamma$ receptor are highly susceptible to attenuated Salmonella Typhimurium [11], our results suggest that colonization is not the major mechanism through which $B$. thuringiensis provides protection against lethal challenge. Rather, other mechanisms such as antimicrobial activity and immune system activation might play major roles in mediating the protective effects of the probiotic B. thuringiensis 87 .

\section{Acknowledgments}

This study was supported by a grant from the Animal, Plant and Fisheries Quarantine and Inspection Agency, Ministry for Food, Agriculture, Forestry and Fisheries, Republic of Korea. This work was also supported in part by a Korea Science and Engineering Foundation (KOSEF) grant funded by the Korean government (MEST) (WCU R33-10045).

\section{References}

1. Abbass A, Sharifuzzaman SM, Austin B. Cellular components of probiotics control Yersinia ruckeri infection in rainbow trout, Oncorhynchus mykiss (Walbaum). J Fish Dis 2010, 33, 31-37.

2. Anadón A, Martínez-Larrañaga MR, Aranzazu Martínez M. Probiotics for animal nutrition in the European Union. Regulation and safety assessment. Regul Toxicol Pharmacol 2006, 45, 91-95.

3. Aronson A. Sporulation and $\delta$-endotoxin synthesis by Bacillus thuringiensis. Cell Mol Life Sci 2002, 59, 417425.

4. Bravo A, Gill SS, Soberón M. Mode of action of Bacillus thuringiensis Cry and Cyt toxins and their potential for insect control. Toxicon 2007, 49, 423-435. 
5. Clinical and Laboratory Standards Institute (CLSI). Performance standards for antimicrobial disk and dilution susceptibility tests for bacteria isolated from animals; approved standards. 2nd ed. NCCLS document M32-A2, CLSI, Wayne, 2002.

6. Doron SI, Hibberd PL, Gorbach SL. Probiotics for prevention of antibiotic-associated diarrhea. J Clin Gastroenterol 2008, 42 (Suppl 2), S58-63.

7. Dunne C, O'Mahony L, Murphy L, Thornton G, Morrissey D, O'Halloran S, Feeney M, Flynn S, Fitzgerald G, Daly C, Kiely B, O'Sullivan GC, Shanahan F, Collins JK. In vitro selection criteria for probiotic bacteria of human origin: correlation with in vivo findings. Am J Clin Nutr 2001, 73 (2 Suppl), 386S-392S.

8. Elmer GW. Probiotics: "living drugs". Am J Health Syst Pharm 2001, 58, 1101-1109.

9. Filho-Lima JVM, Vieira EC, Nicoli JR. Antagonistic effect of Lactobacillus acidophilus, Saccharomyces boulardii and Escherichia coli combinations against experimental infections with Shigella flexneri and Salmonella enteritidis subsp. typhimurium in gnotobiotic mice. J Appl Microbiol 2000, 88, 365-370.

10. Grandy G, Medina M, Soria R, Terán CG, Araya M. Probiotics in the treatment of acute rotavirus diarrhoea. A randomized, double-blind, controlled trial using two different probiotic preparations in Bolivian children. BMC Infect Dis 2010, 10, 253.

11. Hess J, Ladel C, Miko D, Kaufmann SHE. Salmonella typhimurium aroA ${ }^{-}$infection in gene-targeted immunodeficient mice: major role of $\mathrm{CD}^{+}$TCR- $\alpha \beta$ cells and IFN- $\gamma$ in bacterial clearance independent of intracellular location. $\mathrm{J}$ Immunol 1996, 156, 3321-3326.

12. Hong HA, Duc le H, Cutting SM. The use of bacterial spore formers as probiotics. FEMS Microbiol Rev 2005, 29, 813-835.

13. Janssen R, Van Wengen A, Verhard $E$, De Boer $T$, Zomerdijk T, Ottenhoff THM, Van Dissel JT. Divergent role for TNF- $\alpha$ in IFN- $\gamma$-induced killing of Toxoplasma gondii and Salmonella typhimurium contributes to selective susceptibility of patients with partial IFN- $\gamma$ receptor 1 deficiency. J Immunol 2002, 169, 3900-3907.

14. Jones K. Probiotics: preventing antibiotic-associated diarrhea. J Spec Pediatr Nurs 2010, 15, 160-162.

15. Jouanguy E, Döffinger R, Dupuis S, Pallier A, Altare F, Casanova JL. IL-12 and IFN- $\gamma$ in host defense against mycobacteria and salmonella in mice and men. Curr Opin Immunol 1999, 11, 346-351.

16. Kagaya K, Watanabe K, Fukazawa Y. Capacity of recombinant gamma interferon to activate macrophages for Salmonella-killing activity. Infect Immun 1989, 57, 609-615.

17. Kang JH, Yun SI, Park HO. Effects of Lactobacillus gasseri BNR17 on body weight and adipose tissue mass in diet-induced overweight rats. J Microbiol 2010, 48, 712714.

18. Kwon HY, Kim SW, Choi MH, Ogunniyi AD, Paton JC, Park SH, Pyo SN, Rhee DK. Effect of heat shock and mutations in $\mathrm{ClpL}$ and $\mathrm{ClpP}$ on virulence gene expression in Streptococcus pneumoniae. Infect Immun 2003, 71, 3757-3765.

19. Lane DJ. $16 \mathrm{~S} / 23 \mathrm{~S}$ rRNA sequencing. In: Stackebrandt E, Goodfellow M (eds.). Nucleic Acid Techniques in Bacterial Systematics. 1st ed. pp. 115-175, John Wiley \& Sons, Chichester, 1991.

20. Lee DY, Seo YS, Rayamajhi N, Kang ML, Lee SI, Yoo HS. Isolation, characterization, and evaluation of wild isolates of Lactobacillus reuteri from pig feces. J Microbiol 2009, 47, 663-672.

21. Long KZ, Rosado JL, Santos JI, Haas M, Al Mamun A, DuPont HL, Nanthakumar NN, Estrada-Garcia T. Associations between mucosal innate and adaptive immune responses and resolution of diarrheal pathogen infections. Infect Immun 2010, 78, 1221-1228.

22. Marteau PR, de Vrese M, Cellier CJ, Schrezenmeir J. Protection from gastrointestinal diseases with the use of probiotics. Am J Clin Nutr 2001, 73 (2 Suppl), 430S-436S.

23. Nikitenko VI. Infection prophylaxis of gunshot wounds using probiotics. J Wound Care 2004, 13, 363-366.

24. Pineiro M, Stanton C. Probiotic bacteria: legislative framework-- requirements to evidence basis. J Nutr 2007, 137, $850 \mathrm{~S}-853 \mathrm{~S}$

25. Roh JY, Choi JY, Li MS, Jin BR, Je YH. Bacillus thuringiensis as a specific, safe, and effective tool for insect pest control. J Microbiol Biotechnol 2007, 17, 547559.

26. Sleator RD. Probiotics -- a viable therapeutic alternative for enteric infections especially in the developing world. Discov Med 2010, 10, 119-124.

27. van de Vosse E, van Dissel JT, Ottenhoff THM. Genetic deficiencies of innate immune signalling in human infectious disease. Lancet Infect Dis 2009, 9, 688-698.

28. Wilcks A, Smidt L, Bahl MI, Hansen BM, Andrup L, Hendriksen NB, Licht TR. Germination and conjugation of Bacillus thuringiensis subsp. israelensis in the intestine of gnotobiotic rats. J Appl Microbiol 2008, 104, 12521259 . 